\title{
Comment on "Reinterpretation of the thickness-dependent conductivity of thin platinum films"
}

\author{
J. VANCEA, G. REISS, H. HOFFMANN \\ Institut für Angewandte Physik, Universität Regensburg, FRG
}

Messaadi et al. [1] gave a reinterpretation of our data [2] regarding the thickness dependence of the conductivity of platinum films. The conclusion of their letter [1] was that the application of the Fuchs-Namba model [3] to the mentioned results [2] leads to wrong values for the electrical transport parameters, because this model was artificially applied to these films.

The reinterpretation of our experimental data by Messaadi et al. [1] was done using the model published by them $[1,4,5]$ and based only on the replotting of data given in [2] in a $\varrho_{\mathrm{f}}$ (film resistivity) against $1 / d$ ( $d=$ thickness) plot. A linear dependence was established "with exception of two or three experimental points, only". This linear dependence, however, is from our point of view misleading at low thicknesses and can be established only due to the reading errors of the plots given in [1].

In the present comment, we give the original experimental data of the three films under discussion in a $\varrho_{\mathrm{r}}$ against $1 / d$ plot (Figs 1a, 2a and 3a) and in $\varrho_{\mathrm{f}} d$ against $d$ plots (Figs $1 \mathrm{~b}, 2 \mathrm{~b}$ and $3 \mathrm{~b}$ ). The lines I given in the $\varrho_{\mathrm{f}} d$ against $d$ plots (Figs 1a, 2a, 3a) represent the linear fitting approximation of our data made by Messaadi et al. [1], whereas the curves II give the result of the exact Equation 1 of [1]. It should be noted, that this linear approximation (lines I) represents the basis of the discussion, although a serious difference to the exact Equation 1 (lines II) can be stated. Both lines, however, do not follow the experimental data except in Fig. 3a. A simple look at Figs 1 to 3 shows a monotonic deviation of the experimental data from the linear law for these films. The proposed new insight into our experimental data gives the trivial linear dependence for higher and a wrong behaviour for smaller film thicknesses. As intensively discussed in [2], the deviation of the experimental data from the linear behaviour can be explained only by introducing the macroscopic surface roughness (see also [6]).
Here we give a corrective answer to the basic objections made in [1]:

(a) Our fittings to the Namba model are free of any a priori assumptions. Certainly this implies a very high accuracy in experiment and fitting calculations (the suggested accuracy in [1] is not sufficient for this purpose). On the other hand, the model employed by Messaadi et al. [1] neglects totally the background scattering of the conduction electrons at volume defects, i.e. the crystallites are considered free of any defects. This, however, is a rough oversimplification of the well established disordered structure in condensed metallic films. Additionally, the authors assume that the linear law in the experimental $\varrho_{\mathrm{f}}$ against $1 / d$ plot corresponds to the asymptotic expression of Equation 1 in [1] for thick films. Here, it should be noted, that for smaller thicknesses, both the approximation as well as the exact expression diverge completely from the experimental data (see Figs $1 b, 2 b$, $3 b)$. In the absence of any experimental support, this assumption should, therefore, be questioned. However, just a qualitative examination of the experimental $\varrho_{\mathrm{f}} d$ against $d$ curves shows the agreement with the Namba model. Moreover, the roughness determined from electron micrographs of surface replicas of copper and gold films agrees well with the values resulting from fitting calculations (see [7]). Therefore, it seems to be more reasonable to look at the linear law in the $\varrho_{\mathrm{f}} d$ against $d$ (or the $\varrho_{\mathrm{f}}$ against $1 / d$ ) plot as the asymptotic relation of the Fuchs-Namba model.

(b) The examination of Equation 1 in [1] and its asymptotic relation in $[1,3]$ leads to some astonishing facts: in the limit $p=0$ and for the monocrystalline films, the asymptotic relation (Equations 6" and 37 in [3]) leads to:

$$
\frac{\varrho_{\mathrm{f}}}{\varrho_{0}}=1+\frac{3 \lambda_{\mathrm{o}}}{4 d}
$$
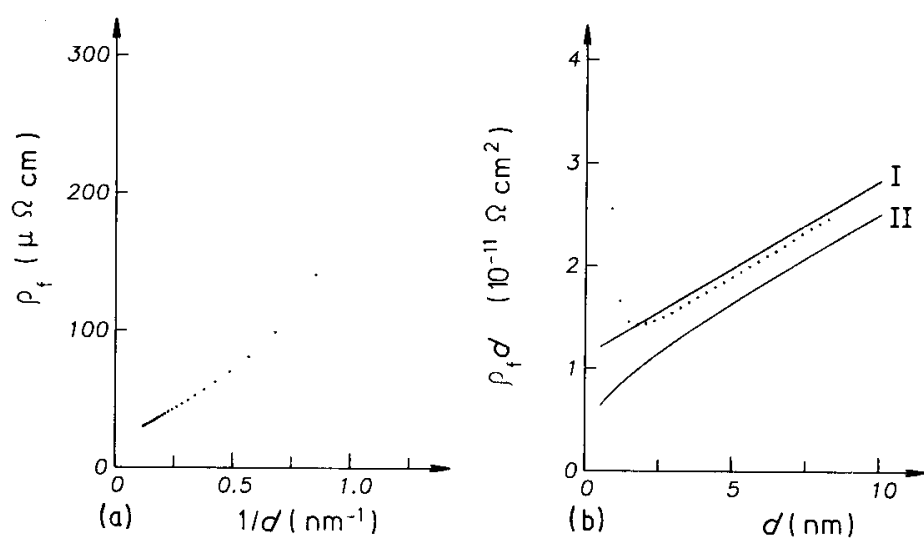

Figure I Sample Pt27 (evaporation rate $0.3 \mathrm{~nm}$ $\sec ^{-1}$ ): (a) $\varrho_{\mathrm{f}}$ against $1 / d$ plot; (b) $\varrho_{\mathrm{f}} d$ against $d$ plot. Points: experimental values. Lines: theoretical expressions of [1] for $p=0.22$ and $t=0.86$. I, linear approximation (Equation 15); II, exact relation (Equation 1). 

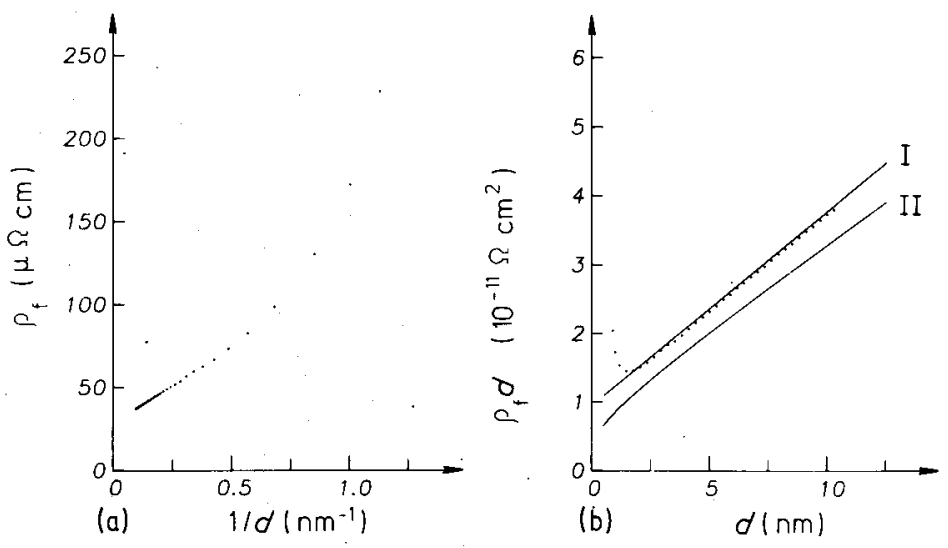

Figure 3 Sample Pt13 (evaporation rate $0.5 \mathrm{~nm}$ $\sec ^{-1}$ ): (a) $\varrho_{\mathrm{f}}$ against $1 / d$ plot; (b) $\varrho_{\mathrm{f}} d$ against $d$ plot. Points: experimental values. Lines: theoretical expressions of [1] for $p=0.69$ and $t=0.68$. I, linear approximation (Equation 15); II, exact relation (Equation 1).
Figure 2 Sample Pt09 (evaporation rate $0.25 \mathrm{~nm}$ $\sec ^{-1}$ ): (a) $\varrho_{\mathrm{f}}$ against $1 / d$ plot; (b) $\varrho_{\mathrm{f}} d$ against $d$ plot. Points: experimental values. Lines: theoretical expressions of [1] for $p=0.3$ and $t=0.61$. I, linear approximation (Equation 15); II, exact relation (Equation 1).
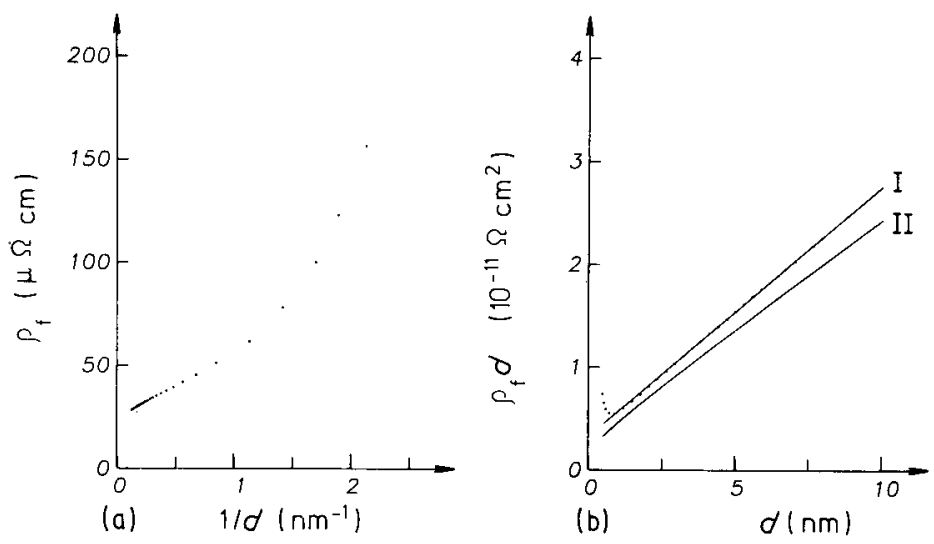

i.e. the size effect is larger by a factor two as given by the Sondheimer approximation. We cannot find, however, any reasons to doubt the validity of the Fuchs theory for monocrystalline films.

Moreover, 'a meaningful theory for polycrystalline films has to fulfil some other criteria. One of them seems to be very clear: if the transmission probability of the electrons at the grain boundaries is zero, the resulting conductivity also has to be zero. This must be fulfilled for any thickness and specularity parameter (see for example MS-theory [8]). Contrary to this fact, Equation 14 in [1] (resistivity $\varrho_{\infty}$ for infinite thick films), leads to $\sigma_{\infty}(t=0) \neq 0$.

(c) The literature cited in [1], unfortunately, is very incomplete. The authors have surely overlooked the paper of Hoffmann et al. [7], where a physical explanation relating to the specularity paramter $(p)$ in platinum films is given. In the same reference, the values of the mfp and the specularity from Namba's model have been confirmed by another, independent experiment (coating experiments on copper films).

In [9], we gave the dependence of the product $\varrho \lambda$ in polycrystalline films for various metals on the number of grain boundaries within one background $\mathrm{mfp}$. Finally, in [10] we proposed a new mechanism of conduction in polycrystalline metals.
Consequently, the arguments of Messaadi et al. [1] relating to the physical consistency of the fitted parameters should be reconsidered.

\section{References}

1. S. MESSAADI, C. R. PICHARD and A. J. TOSSER, J. Mater. Sci. Lett. 5 (1986) 873.

2. H. HOFFMANN and J. VANCEA, Thin Solid Films $\mathbf{8 5}$ (1981) 147.

3. Y. NAMBA, Jpn J. Appl. Phys. 9 (1970) 1326.

4. M. BEDDA, C. R. PICHARD and A. J. TOSSER, $J$. Mater. Sci. 21 (1986) 1405.

5. M. BEDDA, S. MESSAADI, C. R. PICHARD and A. J. TOSSER, ibid. 21 (1986) 2643.

6. K. C. ELSOM and J. R. SAMBLES, J. Phys. F Metal Phys. 11 (1981) 647.

7. H. HOFFMANN, J. VANCEA and U. JACOB, Thin Solid Films 129 (1985) 181.

8. A. F. MAYAdAS and M. ShAtZKes, Phys. Rev. B 1 (1970) 1382

9. J. VANCEA, H. HOFFMANN and K. KASTNER, Thin Solid Films 121 (1984) 201.

10. G. REISS, J. VANCEÁ and H. HOFFMANN, Phys. Rev. Lett. 56 (1986) 2100.

Received 10 February

and accepted 4 March 1987 